\title{
FACTORES QUE INFLUENCIAN LA ADOPCIÓN DE TECNOLOGÍA DE GESTIÓN EN PRODUCCIÓN LECHERA
}

\section{FACTORS INFLUENCING ADOPTION OF DAIRY MANAGEMENT TECHNOLOGY}

\author{
Holmes Rodríguez ${ }^{1 *}$, Carlos J. Ramirez², Fernando Restrepo ${ }^{3}$ \\ Recibido para publicación: Octubre 27 de 2014 - Aceptado para publicación: Mayo 12 de 2015
}

\begin{abstract}
RESUMEN
Antioquia es el departamento de mayor producción de leche de Colombia y la subregión norte representa el $70 \%$ de su producción. No obstante su desarrollo lácteo, subsisten problemas relacionados con la falta de información interna de los sistemas productivos para la toma de decisiones. El objetivo del presente estudio fue evaluar los factores que inciden en la adopción de tecnología de gestión en la producción de leche a partir de un estudio en el municipio de Bello. Se trabajó con un tamaño definitivo de la muestra de 52 fincas, con un nivel de confiabilidad del 95\% y un error máximo permisible del $10 \%$. Se empleó análisis factorial de correspondencia múltiple con clasificación jerárquica indexada, complementado con análisis multivariado de la varianza MANOVA con contraste canónico ortogonal. Se encontró una baja adopción de la tecnología de gestión en la finca lechera por cuanto solo $46 \%$ de los productores maneja registros productivos y se identificaron como factores que inciden en la baja utilización del registro y análisis de la información de la finca para la toma de decisiones la tenencia de la tierra, tamaño del predio, acceso a capacitación, acceso a asistencia técnica, acceso al crédito y tenencia de maquinaria; la composición de la familia no incide, para este contexto, en la adopción de tecnología de gestión.
\end{abstract}

Palabras clave: Asistencia técnica, producción de leche, toma de decisiones.

\begin{abstract}
Antioquia is the province with the highest milk production in Colombia ((70\% of total production). However, some problems associated to lack of information for decision making still remain. The objective of this study was to evaluate the factors influencing adoption of management technology in milk production based on an investigation carried out in Bello, Antioquia. Data from 52 farms were analyzed, . Multiple correspondence indexed factor analysis and multivariate analysis of variance, MANOVA, with canonical orthogonal contrast, were performed. Low adoption of management technology of dairy was found; only $46 \%$ of farmers manage production records. Land tenure, farm size, and low access to training, credit and machinery as well as lack

\footnotetext{
$\overline{1^{*}}$ Ingeniero Agrícola. PhD. Profesor Asistente. Grupo GaMMa, Facultad de Ciencias Agrarias, Universidad de Antioquia UdeA, Calle 70 No. 52-21, Medellín, Colombia. Teléfono (4) 2199133, fax (4) 2199100, holmes.rodriguez@udea.edu.co

${ }^{2}$ Ingeniero Agrónomo. MSc.Grupo GRICA, Facultad de Ciencias Agrarias, Universidad de Antioquia UdeA, Calle 70 No. 52-21, Medellín, Colombia

${ }^{3}$ Estadístico, Especialista en Estadística, Especialista en Biomatemática. Grupo GRICA, Facultad de Ciencias Agrarias, Universidad de Antioquia UdeA, Calle 70 No. 52-21, Medellín, Colombia
} 
of programs of technical assistance for farmers, were the most important factors affecting adoption. The family size did not affect adoption

Key words: technical assistance, milk production, decision making

\section{INTRODUCCIÓN}

En Colombia, la producción de leche es un renglón importante de la economía, con una participación del $10 \%$ en el PIB agropecuario $\mathrm{CNL}$, la cual ha tenido una tasa de crecimiento sostenida en los últimos años, alcanzando en el 2010 una producción de 6363 millones de litros/año. Antioquia por su parte, es el departamento con la mayor producción de leche del país, contribuyendo con un 18,5\% de la producción nacional, alcanzando los 876 millones de litros/año.

En términos generales, el sector lácteo colombiano presenta deficiencias en su competitividad, por lo cual se definió una política nacional para mejorar en este aspecto (CONPES 2010a); en la cual se destaca como uno de los problemas, la baja escolaridad, capacitación y formación de los productores para la toma adecuada de decisiones. A nivel departamental por su parte, la política destaca el bajo nivel de fincas certificadas en buenas prácticas ganaderas; dentro de las cuales, el manejo de registros productivos y reproductivos es un criterio para la certificación.

Si bien es cierto que las políticas para el sector lácteo resaltan la importancia de fortalecer el eslabón productor a través de acciones de acompañamiento técnico y capacitación; la percepción que generalmente tienen pequeños y medianos productores hacia la asistencia técnica dentro de las explotaciones lecheras, según Núñez et al. (2001), está relacionada con un servicio encaminado a resolver problemas netamente técnicos, más no como un medio de información que incide directa e indirectamente en la eficiencia, a partir del manejo adecuado de los recursos. De allí que en el sistema de producción lechero nacional no se maneja la información que permita establecer indicadores productivos.

La falta de información de la finca como soporte para la toma de decisiones, constituye una restricción para el uso eficiente de los recursos productivos; la utilización racional de los recursos implica la utilización de herramientas para el mejoramiento en la toma de decisiones. De acuerdo con estos planteamientos, el manejo de la información se constituye en un insumo fundamental para la toma de decisiones en la empresa agropecuaria; razón por la cual, la carencia de registros, conlleva generalmente a la toma de decisiones con información emanada de percepciones de una realidad sesgada, muchas veces irreal o supuesta.

En ese sentido para Álvarez (2004), la información constituye un recurso esencial para la toma de decisiones, identificando 3 finalidades para su uso, la primera con el fin de actualizar su conocimiento técnico sobre la tecnología de producción, en segundo lugar para entender el ambiente socioeconómico que rodea la agroempresa y finalmente el uso de la información interna para el control del desempeño del sistema productivo. De este modo, se requiere mejorar la adopción por parte de los productores de la tecnología de 
gestión, entendida como el registro y análisis de la información interna para la planificación y la toma de decisiones.

De acuerdo con la literatura, algunos de los factores que inciden en el manejo de la información para la toma de decisiones son el tamaño del predio, la capacitación del personal involucrado en el sistema productivo, el acceso a la asistencia técnica ; la tenencia de la tierra, la pertenencia a grupos y la estructura familiar . Por lo anterior, el objetivo del presente estudio fue identificar factores asociados a la adopción de la tecnología de gestión en la producción de leche por parte de pequeños productores.

\section{MATERIALES Y MÉTODOS}

\section{Técnica de muestreo}

Para llevar a cabo este estudio de tipo descriptivo exploratorio, se utilizó la técnica de muestreo denominada aleatorio de proporciones con factor de ajuste por finitud, teniendo en cuenta un nivel de confiabilidad del 95\% y un error máximo permisible del 10\%, donde los parámetros estimados $\mathrm{P}$ y $\mathrm{Q}$ adoptaron el valor del $50 \%$, debido a que no se poseen estudios anteriores sobre la población objetivo. Con un marco muestral de 456 predios lecheros, el tamaño definitivo de la muestra después de aplicar el factor de corrección por finitud poblacional fue de 52 fincas lecheras.

\section{Técnica de recolección de la información}

Se diseñó una encuesta de tipo estructurado de acuerdo con los objetivos planteados, teniendo en cuenta como variables de tipo cualitativo asociadas a un patrón probabilístico binomial: manejo de registros, tenencia de la tierra, acceso al crédito, acceso a capacitación técnica, acceso a asistencia técnica y tenencia de maquinaria. Como variables cuantitativas de tipo suplementario se tuvieron: tamaño del predio, área en ganadería, altitud y tamaño de la familia. La encuesta se sometió a juicio de expertos en el tema con el objetivo de verificar la pertinencia de las preguntas y de la metodología propuesta y fue aplicada con la participación voluntaria de los productores, a quienes se explicó el objetivo de la investigación.

La base de datos se elaboró en una hoja electrónica de acuerdo con el formato delineado en la encuesta y se realizó un estricto control de calidad en tres etapas del proceso investigativo, con el fin de garantizar los resultados y conclusiones generadas a la luz de la información. Los encuestadores fueron profesionales del sector agropecuario, entrenados para garantizar la confiabilidad en el diligenciamiento de los formatos de recolección de la información.

\section{Análisis estadístico}

Para el análisis estadístico de los datos se empleó análisis factorial de correspondencia múltiple con clasificación jerárquica indexada, teniendo en cuenta como variables activas: manejo de registros, acceso al crédito, acceso a capacitación técnica, acceso a asistencia técnica y tenencia de maquinaria. Anotando que las variables activas se utilizan para realizar el análisis factorial; se utilizó como variable ilustrativa la tenencia o no de la tierra, anotando que esta variable se proyecta sobre la información de las variables activas obtenidas del análisis factorial. Se complementó con el análisis multivariado de la varianza MANOVA con contraste canónico 
ortogonal, estableciendo la dimensionalidad de la comparación multidimensional, por medio del criterio de máxima verosimilitud observando el mayor valor propio significativo, tomando como variable dependiente el manejo de registros y como variables independientes: tamaño del predio, tamaño de la familia, tenencia de la tierra, acceso al crédito, acceso a capacitación técnica, acceso a asistencia técnica y tenencia de maquinaria. El proceso estadístico se complementó por medio de distribuciones de frecuencia de tipo univariado y bivariado mediante tablas de contingencia aplicando la técnica Chi cuadrado. Para el procesamiento de la información fueron utilizados los paquetes estadístico SAS University Edition Virtual Application y SPAD versión 3.5.

Tabla 1. Estadísticas descriptivas unidimensionales.

\section{RESULTADOS Y DISCUSIÓN}

En la zona de estudio en el municipio de Bello, se encontró un tamaño promedio del predio de 16,1 ha, de las cuales el 91,8\% están dedicadas a la ganadería de leche (Tabla 1). Estos resultados indican que la producción de leche es la principal actividad pecuaria de estos productores. Más de la mitad de los productores indagados (54\%) no maneja registros productivos de la finca (Tabla 2), un porcentaje que puede considerarse alto, teniendo en cuenta que el municipio de Bello ha logrado un mayor desarrollo comparativo de su producción láctea respecto a otros municipios del departamento, debido a que su zona de producción se encuentra a menos de $50 \mathrm{~km}$ de la ciudad de Medellín, principal centro de consumo de la región y muy cerca

\begin{tabular}{lc}
\hline \multicolumn{1}{c}{ Variable } & Promedio (n=52) \\
\hline Tamaño del predio (ha) & $16,1 \pm 19,1$ \\
Área en ganadería & $14,8 \pm 13,4$ \\
Altitud (msnm) & $2333,2 \pm 25,4$ \\
Tamaño de la familia (personas) & $3,7 \pm 1,2$ \\
\hline
\end{tabular}

Tabla 2. Análisis descriptivo y comparativo por modalidades asociadas a cada variable.

\begin{tabular}{|c|c|c|}
\hline \multirow{2}{*}{ Variables } & \multicolumn{2}{|c|}{ Porcentaje } \\
\hline & $\mathrm{Si}$ & No \\
\hline Tenencia de tierra & $78,0 \mathrm{a}$ & $78,0 \mathrm{a}$ \\
\hline Acceso al crédito & $38,0 \mathrm{~b}$ & $38,0 \mathrm{~b}$ \\
\hline Acceso a capacitación técnica & $60,0 \mathrm{a}$ & $60,0 \mathrm{a}$ \\
\hline Acceso a asistencia técnica & $58,0 \mathrm{a}$ & $58,0 a$ \\
\hline Tenencia de maquinaria & $62,0 \mathrm{a}$ & $62,0 \mathrm{a}$ \\
\hline
\end{tabular}

*Letras distintas indican diferencia significativa $(\mathrm{p}<0.05)$ 
de las plantas industrializadoras de leche más importantes de la región. Estos resultados indican que en la zona de estudio existe una limitación por parte de los productores para la toma de decisiones por la falta de información; en este sentido, Piñeiro et al. (1998), encontraron que deficiencias en la captura de información puede conducir a problemas de falta de eficiencia en el manejo del sistema productivo.

De los productores indagados, $78 \%$ son propietarios de los predios, lo cual representa una cifra alta, teniendo en cuenta que para la subregión norte, en la cual está localizado el municipio de Bello, el porcentaje de propietarios de los predios alcanza el $63,7 \%$, con un promedio departamental de 56,4\%. El 38\% de los indagados tiene acceso al crédito; los proveedores de crédito son la cooperativa de productores $(38,9 \%)$, la banca comercial (38,9\%), el Banco Agrario (11,1\%) y cooperativas financieras $(11,1 \%)$.

$58 \%$ de los productores indagados cuenta con asistencia técnica; los proveedores de asistencia técnica son la cooperativa de productores $(50 \%)$, asistentes técnicos particulares $(32,1 \%)$, el municipio $(7,1 \%)$, la industria $(3,6 \%)$, proveedor de insumos $(3,6 \%)$ y el propio productor (3,6\%). 60\% de los productores indagados han recibido capacitación técnica; los proveedores de capacitación son la cooperativa de productores $(64,5 \%)$, la industria $(32,3 \%)$ e instituciones públicas $(3,2 \%)$. Se encontró una diferencia altamente significativa $(p<0.0001)$ a favor de los propietarios de la tierra en el manejo de registros; por lo cual para mejorar la adopción de tecnología de gestión deben considerarse estrategias que permitan motivar a quienes carecen de tierra a incorporar el registro $y$ análisis de la información de la finca, como un insumo importante del sistema productivo, para reducir la subjetividad en la toma de decisiones. Este resultado contrasta con los hallazgos de otros autores quienes encontraron que para el contexto de agricultores chilenos, los que arriendan tierras, se sienten más propensos al riesgo y por lo tanto muestran mayor probabilidad a tener información para la toma de decisiones. Se encontró una diferencia altamente significativa $(p<0.0001)$ a favor de los productores con acceso a capacitación técnica en el manejo de registros. Estos resultados concuerdan con los con los hallazgos de Chase et al. (2006), quienes encontraron que para brindar a los productores mejores herramientas en la toma de decisiones, se deben desarrollar programas de capacitación; en este estudio solo $60 \%$ de los productores indagados han sido beneficiarios de capacitación técnica, generalmente en aspectos relacionados con la producción no tanto con el manejo de registros, lo cual representa una limitante para la toma de decisiones por parte de los productores. En este sentido, Avilez et al. (2010) encontraron que los sistemas de producción que llevan registros, obtienen resultados productivos más positivos que aquellos que no lo hacen.

Se encontró una diferencia altamente significativa $(p<0.0001)$ a favor de los productores con acceso a asistencia técnica en el manejo de registros (Tabla 3). Estos resultados concuerdan con los hallazgos de Núñez et al. (2001), quienes encontraron que la ausencia de asistencia técnica se traduce en carencia de registros, convirtiéndose en una limitante para el uso eficiente de los recursos disponibles y para ajustar prácticas de manejo susceptibles de mejorar. 
En este sentido, Cardona y Rodríguez (2005), encontraron que la poca utilización de tecnologías de gestión por parte de los productores, inciden en la sostenibilidad y productividad de los predios; aspecto relacionado con los resultados de Álvarez (2004), en cuanto a la necesidad de mejorar la captura de la información, su análisis, el cálculo básico económico y su uso para la toma de decisiones. Esto sugiere la necesidad de mejorar el acceso de los productores de leche al servicio de asistencia técnica y de capacitación para mejorar la adopción de tecnología de gestión; lo cual implica un mayor compromiso de los actores de la cadena láctea, para facilitar que los productores adquieran habilidades para el registro y análisis de la información de la finca y su uso para la toma de decisiones.
Adicionalmente, se encontró diferencia significativa entre el tamaño del predio y el manejo de registros $(p<0.0001)$. Estos resultados concuerdan con los hallazgos de Piñeiro et al. (1998), quienes encontraron que para el contexto mexicano el tamaño de la finca incide en el manejo de registros. Igualmente se encontró diferencia significativa ( $p>0.0001)$ entre el acceso al crédito y la tenencia de maquinaria con relación al manejo de registros.

Por otro lado, no se encontró diferencia significativa entre el número de integrantes del grupo familiar y el manejo de registros ( $p>0.05)$. Estos resultados contrastan con los hallazgos de Piñeiro et al. (1998) quienes encontraron que la estructura familiar incide en el manejo de registros para el contexto

Tabla 3. Análisis univariado y multivariado de la varianza MANOVA

Prueba

\section{ANAVAS}

ANAVAS

Tamaño del predio

Tamaño de la familia

Tenencia del predio

Acceso al crédito

Tenencia de maquinaria

Acceso a asistencia técnica

Acceso a capacitación

\section{MANOVA}

Wilks' Lambda $<0,0001$

Pillai's Trace

Hotelling-Lawley T

Roy's Greatest R
Valor $\mathbf{P}$

$<0,0001$

$>0,05$

$<0,0001$

$<0,0001$

$<0,0001$

$<0,0001$

$<0,0001$
$<0,0001$ 
mexicano de pequeños, medianos y grandes productores de leche.

El análisis de correspondencia mostró en el primer plano factorial, el cual esta explicado por un $45,7 \%$ de la variabilidad total (Tabla 4) una relación entre los productores que llevan registros y los que poseen asistencia técnica (Figura 1).

Lo anterior sugiere que para mejorar la adopción de tecnología de gestión, es necesario mejorar el acceso de los productores al servicio de asistencia técnica.

Tabla 4. Valores propios y porcentaje acumulado

\begin{tabular}{cccc}
\hline Factor & Valor propio & Porcentaje & Porcentaje acumulado \\
\hline $\mathbf{1}$ & 0,3252 & 27,88 & 27,88 \\
$\mathbf{2}$ & 0,2080 & 17,83 & 45,70 \\
$\mathbf{3}$ & 0,1894 & 16,23 & 61,94 \\
$\mathbf{4}$ & 0,1343 & 11,51 & 73,45 \\
$\mathbf{5}$ & 0,1258 & 10,78 & 84,24 \\
$\mathbf{6}$ & 0,1150 & 9,86 & 94,10 \\
$\mathbf{7}$ & 0,0689 & 5,90 & 100 \\
\hline
\end{tabular}

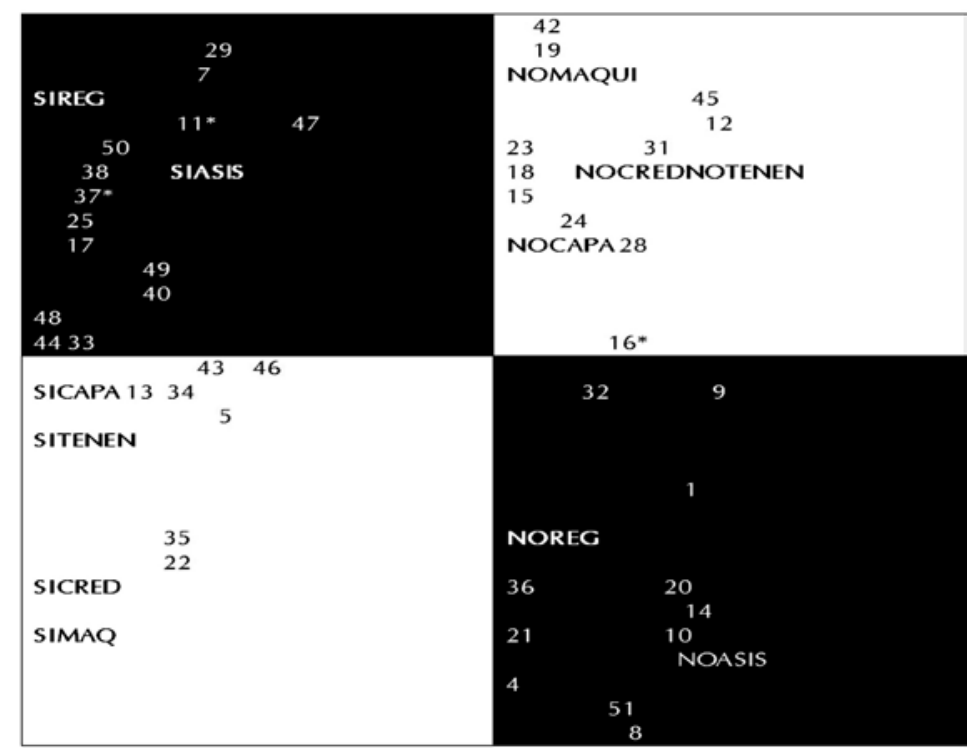

Figura 1. Plano factorial asociado con tenencia de la tierra, maquinaria, asistencia, registros, capacitación y créditos. Los números identifican las fincas,

* indica que existen otras fincas en esa proyección geométrica.

SIREG: Lleva registros; SIASIS: Tiene asistencia técnica; SICAPA: ha recibido capacitación; SITENEN: propietario; SICRED: acceso al crédito; SIMAQ: tiene maquinaria. 
En este sentido es importante resaltar que la cooperativa de productores de la región contribuye con la prestación del servicio de asistencia técnica al 50\% de los productores indagados, lo cual contrasta con la baja participación de los municipios en la prestación de este servicio $(7,1 \%)$, teniendo en cuenta que de acuerdo con la normatividad vigente $y$ por el tamaño promedio de las fincas $(16,1 \mathrm{ha})$ la mayor parte de estos productores se cataloga como pequeños productores, los cuales tienen el derecho al servicio de asistencia técnica gratuita y de obligatorio cumplimiento por parte del municipio.

Se encontró una alta dispersión de las fincas en el análisis de clúster, lo cual indica una gran heterogeneidad entre los grupos de fincas debido básicamente a factores técnicos y económicos representados en la tenencia de la tierra y el acceso al crédito (Figura 2).
Se evidencia claramente tres grupos de fincas, donde el primer grupo de fincas se caracteriza por no tener asistencia técnica, bajo acceso a capacitación y a crédito y bajo manejo de registros. El segundo clúster de fincas se caracterizó por alto acceso a asistencia técnica y capacitación, bajo acceso al crédito y alto manejo de registros. El tercer grupo lo identificaron aquellas fincas con alto acceso a capacitación, asistencia técnica y crédito, alta tenencia de maquinaria propia y mayor tenencia propia de la tierra con alto manejo de registros (Tabla 5), el tamaño de la familia no incidió en la composición de algún clúster en particular.

Este estudio no tuvo en cuenta el nivel educativo del productor, por cuanto de acuerdo con los resultados de Stup et al. (2006), los elementos de gestión que adopte un productor, no parecen estar ligados a su nivel educativo y no serían importantes en

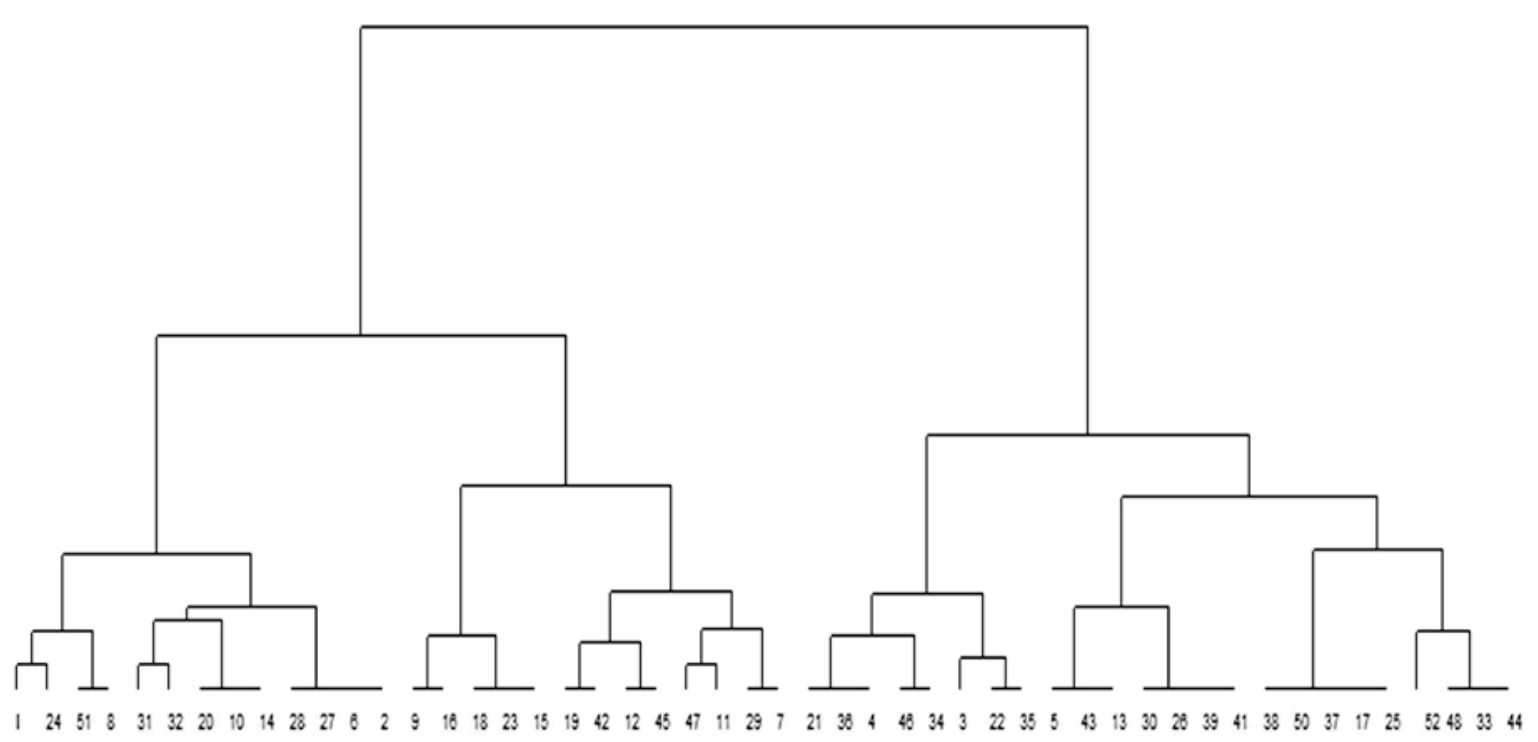

Figura 2. Análisis de clúster 
Tabla 5. Dinámica de las variables en cada conglomerado conformado

\begin{tabular}{lccc}
\hline & Variable & \multicolumn{3}{c}{ Conglomerado } \\
\cline { 2 - 4 } & $\mathbf{1}$ & $\mathbf{2}$ & $\mathbf{3}$ \\
& $(\mathbf{n = 1 3 )}$ & $\mathbf{( n = 1 3 )}$ & $\mathbf{( n = 2 6 )}$ \\
\hline Tamaño del predio (ha) & $11,6 \mathrm{~b}$ & $20,9 \mathrm{a}$ & $16,8 \mathrm{a}$ \\
Área en ganadería (\%) & $99,7 \mathrm{a}$ & $94,0 \mathrm{a}$ & $86,9 \mathrm{~b}$ \\
Tenencia de la tierra (\%) & $69,2 \mathrm{~b}$ & $69,2 \mathrm{~b}$ & $92,3 \mathrm{a}$ \\
Tamaño de la familia & $3,2 \mathrm{a}$ & $4,0 \mathrm{a}$ & $3,8 \mathrm{a}$ \\
Acceso a asistencia técnica (\%) & $23,0 \mathrm{c}$ & $61,5 \mathrm{~b}$ & $77,0 \mathrm{a}$ \\
Acceso a capacitación técnica (\%) & $0 \mathrm{c}$ & $69,2 \mathrm{~b}$ & $88,5 \mathrm{a}$ \\
Acceso al crédito (\%) & $30,8 \mathrm{~b}$ & $15,4 \mathrm{c}$ & $53,9 \mathrm{a}$ \\
Tenencia de maquinaria (\%) & $53,9 \mathrm{~b}$ & $7,7 \mathrm{c}$ & $96,2 \mathrm{a}$ \\
Manejo de registros (\%) & $7,7 \mathrm{~b}$ & $61,5 \mathrm{a}$ & $61,5 \mathrm{a}$ \\
\hline
\end{tabular}

el nivel productivo de su empresa; Piñeiro et al. (1998), sin embargo, encontraron que el nivel educativo del administrador y su familia inciden en el manejo de registros, al igual que otros factores como el tamaño del hato, el ingreso, la pertenencia a grupos y el desarrollo tecnológico. Lo anterior sugiere la necesidad de incluir estos factores en futuros estudios sobre la adopción de tecnología de gestión.

\section{CONCLUSIONES}

La adopción de tecnología de gestión en la producción lechera de la zona estudiada es bajo, convirtiéndose en una limitante para la toma de decisiones objetivas a partir del registro y análisis de la información interna de la finca. Los factores identificados que inciden en esta baja adopción de la tecnología de gestión fueron la tenencia de la tierra, el tamaño del predio, el acceso a capacitación, el acceso a asistencia técnica, el acceso al crédito y la tenencia de maquinaria.

Lo anterior sugiere la necesidad de mejorar la asistencia técnica orientada a facilitar que los productores adquieran habilidades para el registro y análisis de la información de la finca y su uso para la toma de decisiones. La composición de la familia por su parte, no incide, para este contexto, en la adopción de tecnología de gestión.

\section{REFERENCIAS}

Alvarez, J. 2004. La Adopción De Tecnología «Soft » En La Lechería Uruguaya Para El Manejo De La Información, Un Estudio Summary Dairy Farmers ' Information Management and Software Use : a Study Case in Florida, Uruguay. Agrociencia, VIII(1), pp.69-84.

Avilez, et al. 2010. Caracterization productiva de explotaciones lecheras empleando metodología de análisis multivariado. Revista Cientifica-Facultad De Ciencias Veterinarias, 20(1):74-80.

Cardona, C. \& Rodríguez, L. 2005. Análisis de sistema de gestión de la agroempresa 
algodonera del departamento de Córdoba. Agronomia Colombiana, 23(2):342-350.

Chase, L., Ely, L. \& Hutjens, M. 2006. Major advances in extension education programs in dairy production. Journal of dairy science, 89(4):1147-1154.

CNL, 2010. Acuerdo de competitividad de la cadena láctea colombiana. Disponible en: $\quad$ http://www.cnl.org.co/index. php?option=com_remository\&Itemid $=$ 108\&func $=$ startdown \&id $=643$ [Acceso Marzo 29, 2014].

Congreso de Colombia, 2000. Ley 607 de 2000. Disponible en: http://www. secretariasenado.gov.co/senado/ basedoc/ley_0607_2000.html [Acceso Enero 21, 2015].

CONPES, 2010a. Política nacional para mejorar la competitividad del sector lácteo colombiano, CONPES 3675, Bogotá: Departamento Nacional de Planeación, DNP. Disponible en: http://www.cnl.org. co/index.php?option=com_remository \&l temid $=114 \&$ func $=$ download \&id $=640 \& \mathrm{c}$ $\mathrm{hk}=57 \mathrm{fb} 28 \mathrm{a} 0927 \mathrm{a} 51 \mathrm{ca}$ bb7dd34df0619 04d\&no_html=1.

CONPES, 2010b.Unapolítica detransformación productiva: un modelo de desarrollo sectorial para Colombia, CONPES 3678. , p.53. Disponible en: http://www.cnl. org.co/index.php?option $=$ com $_{-}$[Acceso Marzo 29, 2014].
CORLAC, 2012. Acuerdo de competitividad de la cadena láctea de Antioquia Corlac, ed., Medellín. Disponible en: http:// www.establo.info/Documentos/Acuerdo Lacteo Regional Antioquia2012.pdf.

Engler, A. \& Toledo, R., 2010. An analysis of factors affecting the adoption of economic and productive data recording methods of Chilean farmers. Ciencia e investigación agraria, 37(2):101-109.

FEDEGAN, 2010. Producción de leche en Colombia. Disponible en: http:// www.fedegan.org.co/estadisticas/ produccion-0 [Acceso Marzo 18, 2014].

Gobernación de Antioquia, 2012. Política del sector lácteo en Antioquia. Disponible en: http://www.antioquia.gov.co/ Agricultura/Documentos/CRTILLA_ LACTEA.pdf [Acceso Mayo 15, 2015].

MADR, 2007. Agenda Prospectiva de Investigación y Desarrollo Tecnológico de la Cadena Láctea Colombiana. Disponible en: http://www. minagricultura.gov.co/archivos/lacteos. pdf [Acceso Marzo 31, 2015].

MADR, 2010. Oferta Agropecuaria: ENA cifras 2009. , p.194. Disponible en: https://www.agronet.gov.co/www/docs_ [Acceso Abril 15, 2014].

Márquez, M. 2002. La gestión administrativa de las empresas agropecuarias de los municipios San Fernando y Biruaca del Estado Apure en Venezuela. Revista Mexicana de Agronegocios, 6(10):324-335. 
Núñez, J. et al., 2001. El papel de la sistencia Técnica, sobre la productividad y calidad de la leche en las explotaciones lecheras por estado de productor en la región sur del estado de Jalisco. Revista Mexicana de Agronegocios, 5(8), pp.174-180.

Pérez, R. 2000. Sistemas de información para la dirección. Revista Mexicana de Agronegocios, 4(6), pp.467-475.

Piñeiro, D., Chiappe, M. y Graña, F., 1998. La gestión en los establecimientos lecheros, tipologia segun disposición al uso de registros.pdf. Agrociencia, 2(1), pp.125133.
Ruíz, C. 2008. Sustentabilidad financiera: el caso de una empresa ganadera de bovino de doble propósito. Revista Mexicana de Agronegocios, 12(22), pp.503-515.

Stup, R., Hyde, J. \& Holden, L., 2006. Relationships between selected human resource management practices and dairy farm performance. J. Dairy Sci., 89(3), pp.1116-1120.

Trueta, S. 2013. Sistema de información de costos, eficiencia y competitividad (SICEC) de las actividades ganaderas del país (México). Revista Mexicana de Agronegocios, 17(33), pp.622-631. 